\title{
Comparative Analysis of AWG Demultiplexer and Chirped FBG based Demultiplexer in WDM PON Network
}

\author{
Archana Yadav \\ M.Tech. scholar, Department of \\ Electronics \& Communication \\ SSET, SHIATS Allahabad, \\ India
}

\author{
A.K. Jaiswal \\ Prof. \& H.O.D, Department of \\ Electronics \& Communications \\ SSET, SHIATS Allahabad, \\ India
}

\author{
Navendu Nitin \\ Assistant Prof., Department of \\ Electronics \& Communication \\ SSET, SHIATS Allahabad, \\ India
}

\begin{abstract}
In Wavelength Division Multiplexing based Passive Optical Network (WDM-PON), multiple wavelength used to separate Optical Network Unit (ONU). Which actually increase the capacity of PON .WDM process can be dense and ultra-dense depending on channel spacing. In this paper we compare performance of Array Waveguide Grating Demultiplexer (AWG DEMUX) and Fiber Bragg Grating based Demultiplexer (FBG DEMUX) in dense and ultra-dense $\mathrm{WDM}$ at the data rate of $2.5 \mathrm{gbps}$. Optical fiber length used is $10 \mathrm{~km}$. The maximum and minimum Q factor is compare and discussed. All the simulation were performed in optisystem 12.0.
\end{abstract}

\section{General Terms}

Quality Factor, BER, Eye Diagram.

\section{Keywords}

Passive Optical Network, Wavelength Division Multiplexing, Demultiplexer, Array Wave Guide, Fiber Bragg Grating, Ultra Dense and Dense Wavelength Division Multiplexer.

\section{INTRODUCTION}

A Passive Optical Network (PON) is called "passive" because it eliminates "active" electronics in the network. Eliminating these active devices in the network increases the reliability of the network while reducing the amount of maintenance required. PON provide higher bandwidth than traditional copper based access network but it needed further increase in bandwidth of PON by employing WDM so that multiple wavelength may be supported in either up or both up/ downstream direction. Such a PON known as WDM-PON. A WDM-PON provide scalability because it can support multiple wavelength over same fiber infrastructure, is inherently transparent to channel bit rate and does not suffer power splitting losses. Dense WDM (DWDM) has been developed to transmit many wavelength in limited region. DWDM provide channel spacing of $100-200 \mathrm{GHz}$ between successive users. Ultra Dense WDM (UDWDM) technique further decrease the channel spacing to $25-50 \mathrm{GHz}$ which increase the capacity of existing DWDM network. Many users with different wavelength are supported using multiplexer (MUX)/ de-multiplexer (DEMUX). These devices are important part of network. Commercial systems available with capacities of 32 channels and upwards. AWG has become increasing popular as a wavelength multiplexer (MUX) and de-multiplexer (DEMUX). These devices are capable of multiplexing or demultiplexing a large number of wavelengths into a single optical fiber with negligible crosstalk, thereby increasing the transmission capacity of optical networks. AWG De-multiplexer splits optical signal of different wavelength for use in WDM system. The heart of device is AWG which consists of a number of arrayed channel waveguides. The grating offers high wavelength resolution to attaining narrow wavelength channel spacing. The AWGs are also used to multiplex channels of several wavelengths onto a single optical fiber at the transmission end of an optical communication network. A Fiber Bragg Grating (FBG) is a distributed Bragg reflector in optical fiber that reflects particular wavelengths of light and transmits all others by creating a periodic variation in the refractive index of the fiber core, which generates a wavelength-specific dielectric mirror. A fiber Bragg grating can be used in line optical filter to block certain wavelengths, or as a wavelength-specific reflector. They are widely used due to their versatility, cheap fabrication costs and filtering properties. Chirped FBGs can be used for dispersion compensation. Quadratic chirp functions can be implemented when low levels of side lobes are required. The results of Chirped FBG DEMUX is compared with AWG DEMUX.

\section{SIMULATION DESIGN}

The Simulation design of DWDM-PON is shown in figure 1. CW laser array ES of 8 port is used as source with starting frequency $193.1 \mathrm{THz}$. we simulate DWDM by setting frequency spacing to $100 \mathrm{GHz}$ and to simulate UDWDM frequency spacing will be set to $25 \mathrm{GHz}$. The initial phase and line width are kept zero. All ports are fed to the identical subsystems. Internal structure of subsystem is shown in figure 2. PRBS is used to generate bit which is coded by NRZ pulse. MZM with extinction ratio 30 and symmetric factor -1 is used to modulate wavelength. The wavelength is then super imposed by sine generator of frequency $40 \mathrm{GHz}$, amplitude 1 au. and phase angle -90 degree and again modulated by Using MZM with extinction ratio 30 and symmetric factor -1 . Resultant wave is modulated by user defined bit sequence generator. Phase modulator with phase deviation of 90 degree used for modulation. Each subsystem output is multiplexed by WDM MUX ES with starting frequency 193.1 THz. frequency spacing is set $100 \mathrm{GHz}$ to simulate DWDM (To simulate UDWDM frequency spacing kept $25 \mathrm{GHz}$ ). The bandwidth is $10 \mathrm{GHz}$, insertion loss is 0 and the filter type is Bessel of order 2. The multiplexed wave is launched in optical fiber of length $10 \mathrm{~km}$, with reference wavelength of $1550 \mathrm{~nm}$, attenuation of $0.2 \mathrm{~dB} / \mathrm{km}$, and dispersion parameter of 16.75 $\mathrm{ps} / \mathrm{nm} / \mathrm{km}$. The dispersion slop is $.075 \mathrm{ps} / \mathrm{nm} / \mathrm{km}$ and differential group delay is $0.2 \mathrm{ps} / \mathrm{km}$. The input provided passed to the fiber as shown in figure 4. To demultiplexer AWG DEMUX and DEMUX using chirped FBG are used. AWG DEMUX of size 8 is used to demultiplexing the signal out of optical fiber. Configuration is set to DEMUX and frequency spacing to $100 \mathrm{GHz}$ to simulate DWDM. Filter type is Gaussian of order 2. We need optical null to nullify the 
other inputs of AWG. To secure it from any noise. Each demultiplexed signal from AWG is passed through Avalanche photo diode and filtered by low pass Bessel filter of order 4 . The Bit Error Rate (BER) visualizer is used to analyse maximum and min quality factor for each wavelength channel. Eye diagram can also see by BER visualizer. We compare AWG DEMUX result with chirped FBG DEMUX. Chirped FBG based DEMUX is shown in figure 3. The design has each FBG in the DEMUX which reflects one successive wavelength from multiplexed signal. Which are then fed to output port. No need to use circulators. It require only one
FBG for separating each wavelength. This design has great advantage for 16,32 or 64 channel system. This design is relatively cheaper as compared to other FBG DEMUX. In this each FBG have an effective index of 1.2 and all have same length i.e. $35 \mathrm{~mm}$. We choose Gaussian apodization function having Gaussian parameter of 0.2. In compare to tanh function Gaussian was chosen because it gives better spectral response as it was chirp FBG DEMUX, the chirp function is selected to quadratic with quadratic parameter of $0.00015 \mathrm{um}$ which provide great dispersion compensation.

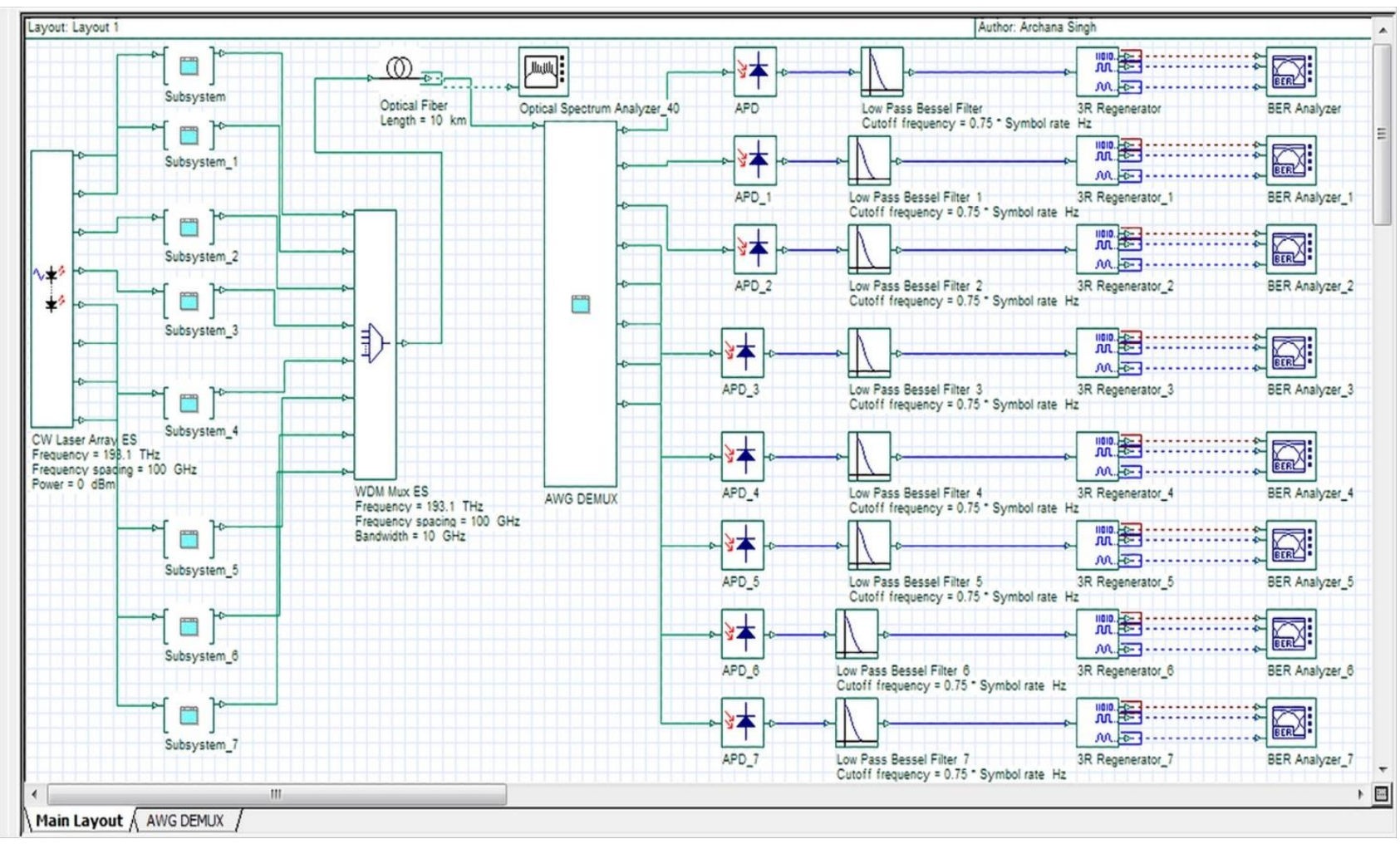

Figure 1. Simulation design of DWDM-PON

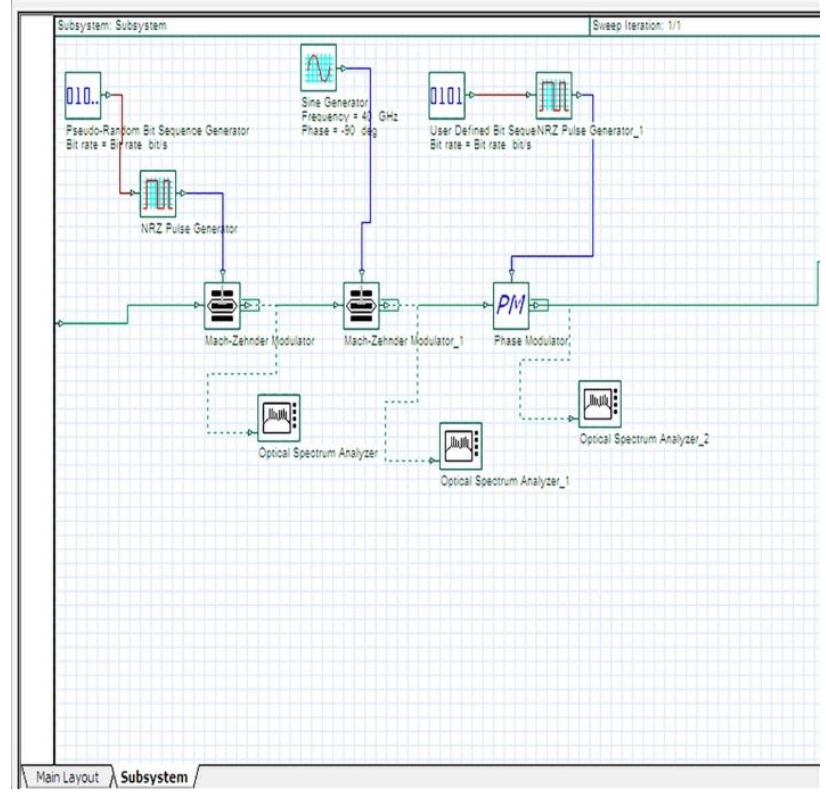

Figure 2. Subsystem Internal Structure

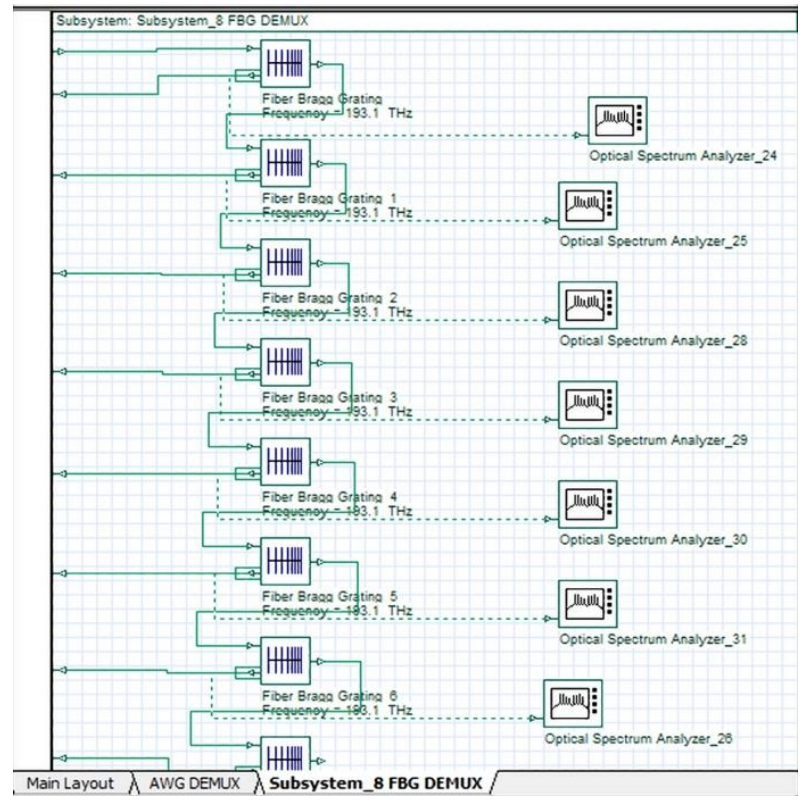

Figure 3. Chirped FBG Demultiplexer 


\section{SIMULATION RESULT AND DISCUSSION}

를 Optical Spectrum Analyzer_40

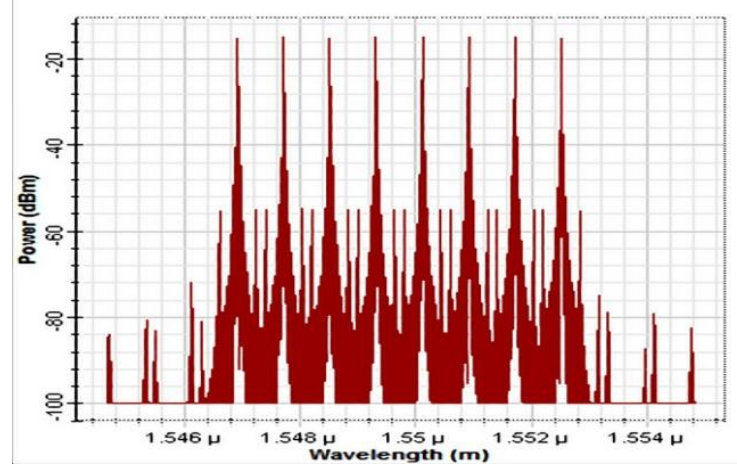

Figure 4: Input provided to fiber

Both AWG DEMUX and chirped FBG DEMUX is simulated in DWDM-PON Network. Output of AWG DEMUX port 1 and port 8 is shown in figure 5. Also output of chirped FBG DEMUX port 1 and port 8 shown in figure 6. In AWG DEMUX output port 1 and port 8 some anomalies were observed. These spurious response also seen in port 2 and port7. These spurious responses was at $193.9 \mathrm{THz}$ for port 1 while at $193.1 \mathrm{THz}$ for port 8 . Other ports 3, 4, 5 and 6 did not show these kind of responses. In FBG DEMUX output, only port 1 and 8 show these responses. Spurious response for port 1 is at $193.8 \mathrm{THz}$ and for port 8 it was at $193.1 \mathrm{THz}$.$$
\text { 魚 }
$$

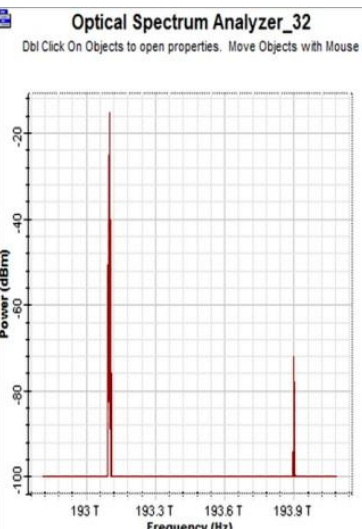

B Optical Spectrum Analyzer 39

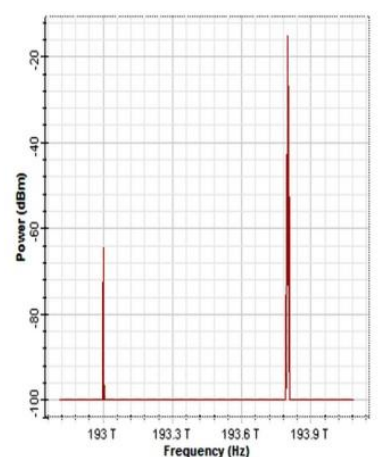

Figure 5: Output of AWG DEMUX Port 1 and 8 spurious response.

$$
\text { 急 }
$$

Optical Spectrum Analyzer_24 Dol cick On Objects to open propenties. Move Objects wath Mous or

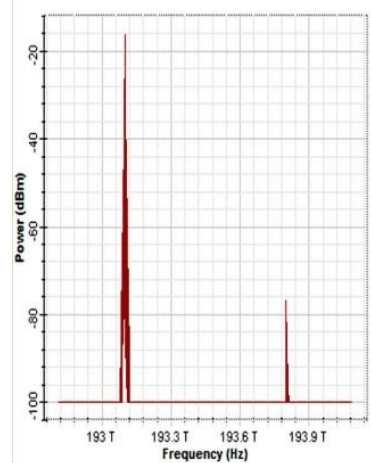

: Optical Spectrum Analyzer_27 Dob cick On Objects to open properties. Move Objects weth Mouse Orag

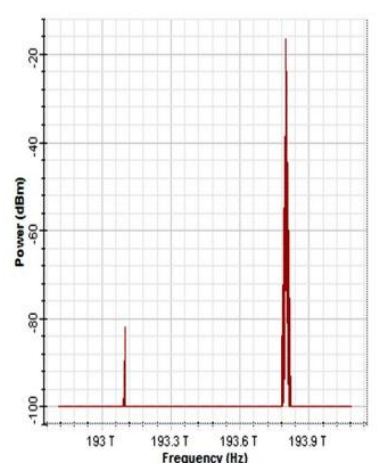

Figure 6: Output of FBG DEMUX Port 1 and 8 also show spurious response
The max and min Q factor for UDWDM (frequency spacing $25 \mathrm{GHz}$ ) and DWDM (frequency spacing 100GHz) using AWG DEMUX and chirped FBG DEMUX tabulated in table: Table 1

\begin{tabular}{|l|l|l|}
\hline Frequency spacing & $\begin{array}{l}\text { AWG } \\
\text { DEMUX }\end{array}$ & $\begin{array}{l}\text { Chirped FBG } \\
\text { DEMUX }\end{array}$ \\
\hline 25GHz(UDWDM) & $\begin{array}{l}\text { Max: } \\
150.463 \\
\text { Min: } \\
\end{array}$ & Max: 155.867 \\
& 110.681 & \\
\hline $100 \mathrm{GHz}(\mathrm{DWDM})$ & Max: 115.281 \\
& 151.928 & Max:152.456 \\
& Min: & \\
& 120.167 & \\
\hline
\end{tabular}

It can see from table 1 that for UDWDM, AWG and FBG DEMUX both gives approximate similar $\max$ and $\min \mathrm{Q}$ factor but for DWDM, AWG provide better max min range than FBG DEMUX. FBG DEMUX, for DWDM have long interval between maximum and minimum range. Figure 7 and 8 show maximum and minimum $\mathrm{Q}$ factor diagram for both AWG and FBG respectively for DWDM. The eye diagram at max and min Q factor for AWG DEMUX is clear and wide open. While the eye diagram at min $\mathrm{Q}$ factor for FBG DEMUX is little blurred.
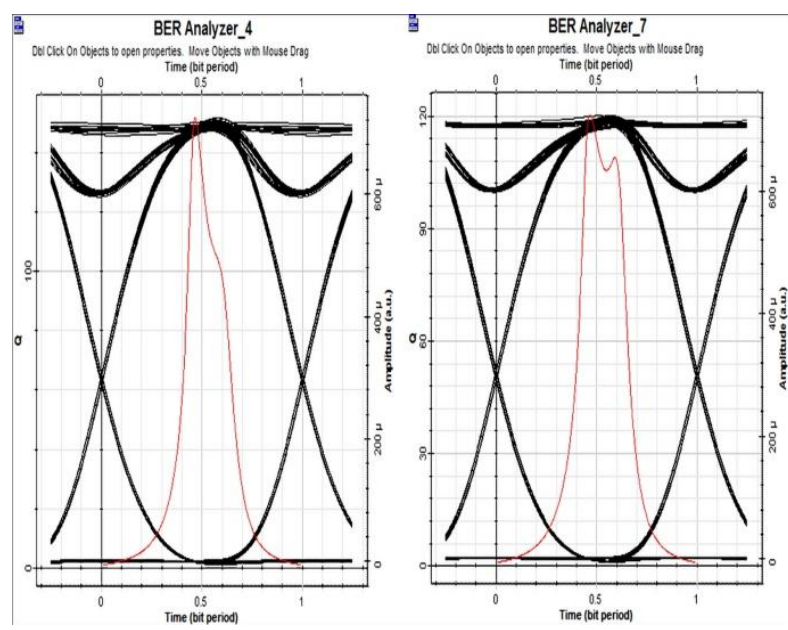

Figure 7: Max and Min Q factor for AWG DEMUX
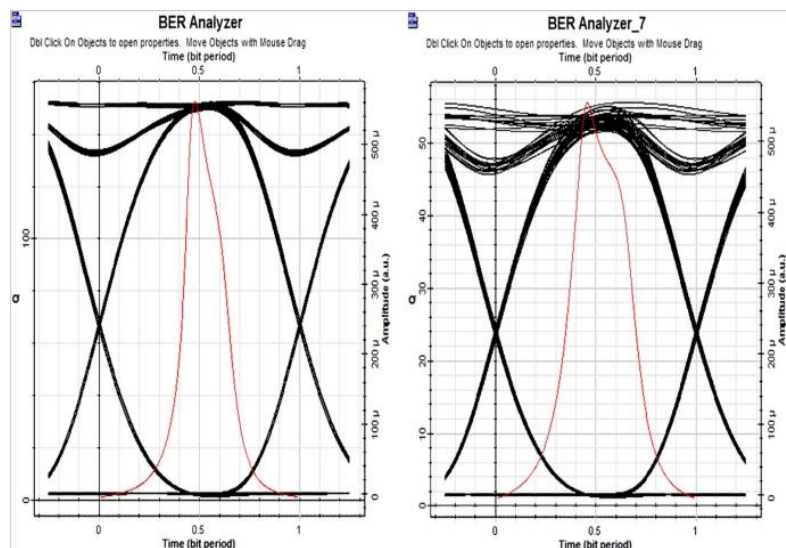

Figure 8: Max and Min Q factor for chirped FBG DEMUX 


\section{CONCLUSION AND FUTURE SCOPE}

The performance comparison of AWG DEMUX and chirped FBG DEMUX was performed with frequency spacing of 25 $\mathrm{GHz}$ and $100 \mathrm{GHz}$ in order to simulate DWDM and UDWDM respectively. The design was simulated at the data rate $2.5 \mathrm{gbps}$ and length of fiber was $10 \mathrm{~km}$. the comparison of output of both DEMUX for port 1 and 8 is discussed. It was observed that overall chirped FBG DEMUX in UDWDM PON gives great maximum $Q$ factor compare to DWDM. Which means frequency spacing of $25 \mathrm{GHz}$ is more efficient than $100 \mathrm{GHz}$ as frequency spacing between DEMUX wavelengths. And in AWG DEMUX max $Q$ factors are approx. similar to each other for UDWDM and DWDM.

\section{REFERENCES}

[1] Amitabha Banerjee, November 2005, "Wavelengthdivision-multiplexed passive optical network (WDMPON) technologies for broadband access: a review, journal of optical networking, vol. 4, p. 11.

[2] D. Seyringer et al. November, 2012, "Design, Simulation and Evaluation of AWG Based Demultiplexers", the Ninth International Conference on Advanced Semiconductor Devices and Microsystems.

[3] Giles et al., November 1996, "Access PON Using Downstream 1550-nm WDM Routing and Upstream 1300-nrn SCMA Combining Through a Fiber-Grating Router", IEEE photonics technology letters, vol. 8, no 11.

[4] Guido Maier et al., February 2011, "Design and Cost Performance of the Multistage WDM-PON Access Networks", journal of lightwave technology, vol. 18, no.2.
[5] Jiajia Chen, Lena Wosinska, Mohsan Niaz Chughtai, and Marco Forzati, September 2011, "Scalable Passive Optical Network Architecture for Reliable Service Delivery", optical communication network, vol 3, no.9.

[6] J.A.R. Williams, I. Bennion, K. Sugden, N. J. Doran, 1994, "Fiber dispersion compensation using a chirped in fiber Bragg grating”, Electronics Letter, Vol. 30, p.985987.

[7] J.R. Stern et al., 1988, "Passive optical networks for telephony applications and beyond", Electronics Letter, Vol. 23, p. 1255-1257.

[8] Karan Gupta, Taranand Mukhopadhyay, Abhishek Goyanka, December 2013, "Design and Simulation Chirped fiber Bragg Grating Based Demultiplexer for Ultra-Dense Wavelength Division Multiplexing Based Passive Optical Network" Advanced Networks and Telecommunications Systems (ANTS) IEEE International Conference.

[9] R. Romero et al., 2003, "Multiplexer and Demultiplexer using chirped Fiber Bragg Gratings and optical circulators" , High Speed Networks and Multimedia Communications Lecture Notes in Computer Science, Vol. 2720, pp 442-451.

[10] T. Erdogan, 1997, "Fiber Grating Spectra", J. Lightwave Technology, vol. 15, p. 1277-1294.

[11] V. Mizrahi et al., 12 May 1994, "Four Channel Fiber Grating Demultiplexer", IEEE Electronics Letters, Vol. 30, No. 10 . 